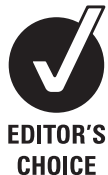

CHOICE

\title{
H1N1 vaccines in a large observational cohort of patients with inflammatory bowel disease treated with immunomodulators and biological therapy
}

\author{
Jean-François Rahier, ${ }^{1}$ Pavol Papay, ${ }^{2}$ Julia Salleron, ${ }^{3}$ Shaji Sebastian, ${ }^{4}$ \\ Manuela Marzo, ${ }^{5}$ Laurent Peyrin-Biroulet, ${ }^{6}$ Valle Garcia-Sanchez, ${ }^{7}$ Walter Fries, ${ }^{8}$ \\ Dirk P van Asseldonk, ${ }^{9}$ Klaudia Farkas, ${ }^{10}$ Nanne K de Boer, ${ }^{11}$ Taina Sipponen, ${ }^{12}$ \\ Pierre Ellul, ${ }^{13}$ Edouard Louis, ${ }^{14}$ Simon T C Peake, ${ }^{15}$ Uri Kopylov, ${ }^{16}$ Jochen Maul, $^{17}$ \\ Badira Makhoul, ${ }^{18}$ Gionata Fiorino, ${ }^{19}$ Yazdan Yazdanpanah, ${ }^{20}$ Maria Chaparro, ${ }^{21}$ for \\ the European Crohn's and Colitis Organisation (ECCO)
}

\section{See Commentary, p 433 \\ For numbered affiliations see end of article.}

\section{Correspondence to \\ Dr Jean-François Rahier, Service d'Hépatogastroentérologie, Cliniques Universitaires UCL Mont-Godinne, 1 avenue Dr Therasse, 5530 Yvoir, Belgium; jfrahier@gmail.com}

J-FR and PP contributed equally to the article.

Revised 17 December 2010 Accepted 21 December 2010 Published Online First 26 January 2011

\section{ABSTRACT}

Background Safety data are lacking on influenza vaccination in general and on $\mathrm{A}(\mathrm{H} 1 \mathrm{~N} 1)$ v vaccination in particular in patients with inflammatory bowel disease (IBD) receiving immmunomodulators and/or biological therapy.

Aims and methods The authors conducted a multicentre observational cohort study to evaluate symptoms associated with influenza H1N1 adjuvanted (Pandemrix, Focetria, FluvalP) and non-adjuvanted (Celvapan) vaccines and to assess the risk of flare of IBD after vaccination. Patients with stable IBD treated with immunomodulators and/or biological therapy were recruited from November 2009 until March 2010 in 12 European countries. Harvey-Bradshaw Index and Partial Mayo Score were used to assess disease activity before and 4 weeks after vaccination in Crohn's disease (CD) and ulcerative colitis (UC). Vaccination-related events up to 7 days after vaccination were recorded.

Results Of 575 patients enrolled (407 CD, 159 UC and nine indeterminate colitis; $53.9 \%$ female; mean age 40.3 years, SD 13.9), local and systemic symptoms were reported by $34.6 \%$ and $15.5 \%$ of patients, respectively. The most common local and systemic reactions were pain in $32.8 \%$ and fatigue in $6.1 \%$ of subjects. Local symptoms were more common with adjuvanted (39.3\%) than non-adjuvanted $(3.9 \%)$ vaccines $(p<0.0001)$, whereas rates of systemic symptoms were similar with both types (15.0\% vs $18.4 \%, p=0.44)$. Among the adjuvanted group, Pandemrix more often induced local reactions than FluvalP and Focetria (51.2\% vs $27.6 \%$ and $15.4 \%, p<0.0001$ ). Solicited adverse events were not associated with any patient characteristics, specific immunomodulatory treatment, or biological therapy. Four weeks after vaccination, absence of flare was observed in 377 patients with CD (96.7\%) and 151 with UC (95.6\%). Conclusion Influenza A (H1N1)v vaccines are well tolerated in patients with IBD. Non-adjuvanted vaccines are associated with fewer local reactions. The risk of IBD flare is probably not increased after $\mathrm{H} 1 \mathrm{~N} 1$ vaccination.

\section{INTRODUCTION}

In April 2009, the Center for Disease Control and Prevention identified two cases of human infection with influenza A (H1N1)v characterised by a unique combination of gene segments that had

\section{Significance of this study}

What is already known about this subject?

- Patients with inflammatory bowel disease (IBD) treated with immunomodulators and biological therapy are a fragile population.

- Protection against influenza disease is achieved by vaccination in immunocompetent individuals. For immunocompromised patients (the study sample described here), data for clinical protection from infection are lacking. Antibody seroconversion response is variable, with some studies suggesting good immunogenicity and others showing poor response.

What are the new findings?

- The types of adverse events after influenza $A$ H1N1 vaccination in patients with IBD are similar to those reported in the immunocompetent population.

- Solicited adverse events after influenza A H1N1 vaccination are not related to type of IBD or type of immunodulators and biological therapy.

- Adjuvanted vaccines induce more local symptoms that non-adjuvanted vaccine in patients with IBD receiving immunomodulatory and biological therapy.

- In a very large majority of patients, adjuvanted and non-adjuvanted influenza vaccines do not affect IBD activity.

How might it impact on clinical practice in the foreseeable future?

- This study brings new evidence on the good safety profiles of adjuvanted and non-adjuvanted influenza vaccines in the immunocompromised IBD population and indicates that such vaccines are unlikely to induce flare up in patients with IBD. It is therefore a very important message in case of future pandemics. not been identified in human influenza A virus. ${ }^{1}$ Additional cases were rapidly reported, leading WHO to declare a pandemic phase level 6 , indicating widespread human infection. This caused 
anxiety, especially among physicians and patients who were potentially immunocompromised. Prevention of influenza is usually achieved by vaccination, and new vaccines directed towards the influenza A (H1N1)v infection were manufactured. Two larges studies have produced reassuring data on the efficacy and safety of these novel vaccines, whether in an MF59-adjuvant form or not, in young and middle-aged adults. ${ }^{2} 3$ These data are difficult to extrapolate to other categories of people, particularly adults who have underlying immune suppression, which is the group for whom the influenza $\mathrm{A}(\mathrm{H} 1 \mathrm{~N} 1) \mathrm{v}$ vaccine is particularly recommended. Patients with inflammatory bowel disease (IBD) belong to a younger population, are affected with a chronic immune-mediated inflammatory disorder, and are immunocompromised to some extent when immunomodulators such as corticosteroids, methotrexate, thiopurines and antitumour necrosis factor agents are used. Because of this immunosuppression, national and international recommendations and expert advice have emphasised the need to vaccinate patients with IBD and immunocompromised conditions against influenza infection. ${ }^{4}$ However, data on the global safety and efficacy of adjuvanted and non-adjuvanted vaccines are lacking in patients with IBD.

We conducted a European multicentre prospective study to (i) evaluate unsolicited and solicited local and systemic symptoms associated with influenza H1N1 vaccination in patients with IBD receiving immmunomodulatory and/or biological therapy, (ii) compare rates and types of unsolicited and solicited local and systemic symptoms in adjuvanted and non-adjuvanted H1N1vaccines, (iii) assess the risk of flare of IBD following H1N1 vaccination, and (iv) search for factors associated with increased adverse events or disease flare.

\section{METHODS}

\section{Study design}

We conducted a multicentre, prospective cohort study from November 2009 through March 2010 in 12 European countries. Patients with IBD were recruited consecutively during outpatient visits in 24 academic and non-academic IBD centres. They were eligible if they had a stable inflammatory disease at the time of the visit (left to the physician discretion), they were treated with one or more immunomodulator and/or biological therapy, and a single- or two-dose vaccination for $\mathrm{H} 1 \mathrm{~N} 1$ virus was planned according to national recommendations. A stable disease was defined as one without signs of activity (biological, endoscopic or clinical) and not requiring any treatment modification for the IBD. The only exclusion criteria were an active inflammatory disease (left to the physician discretion). The type of vaccine was decided by the national health authorities. The vaccine was administered by intramuscular injection according to the manufacturer's recommendations either by the general practitioner or in a government vaccination centre. Patients who had received the $\mathrm{H} 1 \mathrm{~N} 1$ vaccine before the outpatient visit were not included. Validated clinical activity indexes-HarveyBradshaw Index $(\mathrm{HBI})^{5}$ and Partial Mayo Score (PMS) ${ }^{6}$-were used for Crohn's disease (CD) and ulcerative colitis (UC), respectively, to assess disease activity (boxes 1 and 2). Data from each patient were collected using a standardised questionnaire. During the outpatient visit and before vaccination, all patients were scored. Four weeks after the last dose of H1N1vaccine, patients were contacted by phone to assess the IBD clinical activity index and the presence of unsolicited and solicited local (pain, redness, warmth, swelling) and systemic (shivering, fever $>38^{\circ} \mathrm{C}$, unusual fatigue, malaise, headache, muscle pain, arthralgia, use of pain killers) symptoms occurring within 7 days
Box 1 Harvey-Bradshaw Index, a simple index of Crohn's disease activity

A. General well-being:

$0=$ very well

$1=$ slightly below par

$2=$ poor

$3=$ very poor

$4=$ terrible

B. Abdominal pain:

$0=$ none

$1=$ mild

2 = moderate

$3=$ severe

C. Number of liquid stools per day:

D. Abdominal mass:

$0=$ none

$1=$ dubious

2 = definite

3 = definite and tender

E. Complications: (score 1 per item)

Arthralgia

Uveitis

Erythema nodosum

Aphthous ulcers

Pyoderma gangrenosum

Anal fissure

New fistula, abscess

of vaccination. Symptoms were considered to be present or absent. As in clinical trials, ${ }^{2}$ unsolicited and solicited events were considered vaccine-related if occurring within 7 days of

Box 2 Partial Mayo Score, a scoring system for assessment of ulcerative colitis activity

Stool frequency*

$0=$ normal number of stools for this patient

$1=1-2$ stools more than normal

$2=3-4$ stools more than normal

$3=5$ or more stools than normal

Rectal bleedingt

$0=$ no blood seen

$1=$ streaks of blood with stool less than half the time

$2=$ obvious blood with stool most of the time

$3=$ blood alone passed

Physician's global assessment

$0=$ normal

$1=$ mild disease

2 = moderate disease

$3=$ severe disease

* Each patient served as his or her own control to establish the degree of abnormality of the stool frequency.

†The daily bleeding score represented the most severe bleeding of the day.

¥The physician's global assessment acknowledged the three other criteria, the patient's daily record of abdominal discomfort and general sense of well-being, and other observations, such as physical findings and the patient's performance status. 
vaccination. Adverse reactions were defined as any reaction that persisted beyond 7 days after vaccination. Serious adverse reactions were defined as any reaction that necessitated hospitalisation. Approval for the study protocol was obtained from the central and local ethics committees, and the study was conducted in accordance with the principles of the Declaration of Helsinki. Written informed consent was obtained from each subject. The study was scientifically supported by the European Crohn's and Colitis Organisation (ECCO).

\section{Vaccines}

Four vaccines directed against the $2009 \mathrm{H} 1 \mathrm{~N} 1$ virus were used in the study. All vaccines were non-live and licensed in the European Union at the time of the pandemic. Three vaccines were adjuvanted (Pandemrix, Focetria and FluvalP) and one was non-adjuvanted (Celvapan). According to manufacturers' and national recommendations, either a single injection or two injections were administered to the patients. The composition of the vaccines differed as follows:

Pandemrix: one dose $(0.5 \mathrm{ml})$ contains split influenza virus, inactivated, containing $3.75 \mu \mathrm{g}$ antigen equivalent to $\mathrm{A} / \mathrm{Cali}$ fornia/7/2009 (H1N1)v-like strain (X-179A); AS03 adjuvant composed of squalene (10.69 mg), DL- $\alpha$-tocopherol (11.86 mg) and polysorbate $80(4.86 \mathrm{mg}) ; 5 \mu \mathrm{g}$ thiomersal (excipient).

Focetria: one dose $(0.5 \mathrm{ml})$ contains $7.5 \mu \mathrm{g}$ influenza virus surface antigens of strain A/California/7/2009 (H1N1)v-like strain (X-181); adjuvant MF59C.1 containing squalene $9.75 \mathrm{mg}$, polysorbate $801.175 \mathrm{mg}$ and sorbitan trioleate $1.175 \mathrm{mg}$.

FluvalP: one dose $(0.5 \mathrm{ml})$ contains $6 \mu \mathrm{g}$ whole virion, reassortant A/California/7/2009 (H1N1)v-like strain and adjuvant aluminium phosphate gel.

Celvapan: one dose $(0.5 \mathrm{ml})$ contains whole virion influenza vaccine, inactivated, containing $7.5 \mu \mathrm{g}$ antigen of pandemic strain A/California/07/2009 (H1N1)v.

\section{Patient characteristics, side effects and disease activity}

Clinical data included age, sex, pregnancy, disease duration, IBD phenotype according to the Montreal classification, ${ }^{7}$ type and dosage of the immunomodulator and biological therapy, unsolicited and solicited local and systemic symptoms. A solicited adverse record is derived from organised data collection systems, whereas an unsolicited adverse event is any adverse event spontaneously reported or reported after questioning. Combined therapy was defined as the concomittant use of two or more drugs (immunomodulators and/or biological therapy). All selfreported reactions were recorded independently of their severity. Number of bowel movements a day and onset of diarrhoea or bloody diarrhoea within 7 days of vaccination were recorded. Disease activity at baseline (before vaccination) and 4 weeks after vaccination was assessed by the HBI and PMS in CD and UC patients, respectively. Absence of flare was defined as either decreased, unchanged or increased (by a maximum of 2 points) clinical activity index score. For both UC and CD patients, an increase of 3 points or more observed 4 weeks after vaccination was considered to be a clinical flare according to the literature. $^{8-11}$ Additional vaccinations with seasonal influenza vaccine and pneumococcal polysaccharide vaccine were recorded. Dates of all vaccinations and events were noted.

\section{Statistical analysis}

Patients with incomplete data were not included in the analysis. All statistical analysis was performed using SAS software V.9.1. Continuous data are expressed as mean (SD) or median (IOR) when appropriate. Qualitative data are expressed as frequency and percentage. $\mathrm{p}<0.05$ was considered significant. To study the relation between qualitative variables, $\chi^{2}$ test or Fisher exact test was performed. The difference in a continuous variable according to a binary variable was studied using the Student $t$ test. Variables with $p<0.2$ were introduced into a stepwise multivariate logistic regression (significance level for entering effects, 0.2; significance level for removing effects, 0.05). The stability of the model was assessed by a bootstrap method. ${ }^{12}$ The bootstrap resampling method was based on 500 replicates of the initial dataset. Logistic multivariate regression with stepwise selection at the 0.2 level was performed on each of these replicates. The inclusion of the variable in the final model was confirmed if this candidate variable was selected in at least $80 \%$ of these 500 analyses.

\section{RESULTS}

Demographic, clinical characteristics and immunomodulatory treatments of patients are given in table 1 . Of 733 patients who were eligible and signed an informed consent, 124 decided not to receive the vaccine. A total of 609 subjects who were administered the $\mathrm{H} 1 \mathrm{~N} 1$ vaccines were enrolled in the study, but complete data were available for 575 subjects. An adjuvanted vaccine was given to 499 patients, and a non-adjuvanted vaccine to 76. A second-dose H1N1 vaccine was given to 212 patients (66.0\% and $34.0 \%$ in the adjuvanted and non-adjuvanted group, respectively) separated by a median of 23 days. The mean age of the subjects was 40.3 years (SD 13.9); $53.9 \%$ were women, and six were pregnant. Of the 575 subjects, 407 had CD, 159 had UC, and nine had indeterminate colitis. Median disease duration was 9 years for CD (IOR 4.2-15), 7 years for UC (IOR 4-13), and 3.5 year for indeterminate colitis (IOR 3-5). All patients were treated with one or more immunomodulators or biological therapy. Of the 575 patients, $41.7 \%$ were given monotherapy and $58.3 \%$ received combined therapy. Seasonal influenza vaccine was given additionally to 328 patients (57.0\%), $68.8 \%$ of them $(n=196)$ before the H1N1 vaccine (median 28 days; IOR $15-42.5)$ and $23.1 \%(n=66)$ on the same day; $8.1 \%$ received seasonal vaccination after the $\mathrm{H} 1 \mathrm{~N} 1$ vaccine (median 25 days; IOR 11-31). Pneumococcal vaccine was administered in $12.2 \%$ $(n=70)$ of all patients. The baseline characteristics were similar among patients with adjuvanted and non-adjuvanted vaccines except for the use of infliximab, the number of H1N1 injections, the presence of perianal disease, and the use of pneumococcal vaccine (table 1 ).

\section{Safety analysis}

Solicited local and systemic reactions

Data from all 575 patients were included in safety analyses. Solicited local and systemic reactions during the first 7 days after the first dose of H1N1 vaccine are shown in table 2. Local and systemic solicited reactions appeared mostly on the day after vaccination, and disappeared within $48-72 \mathrm{~h}$. No unsolicited events, adverse reactions or serious adverse reactions were reported.

The most common local reaction after any type of vaccine was pain at the injection site, reported by 189 (32.8\%) subjects. Swelling, redness and warmth was present in $35(6.1 \%), 30$ $(5.2 \%)$ and $10(1.7 \%)$ patients, respectively. With the same subtype of vaccine, patients with CD and UC had similar rates of local symptoms. No severe local reactions were reported. Pain was more common after injection of the adjuvanted vaccine than with non-adjuvanted vaccine $(37.3 \%$ vs $3.9 \%, p<0.0001)$. Among the adjuvanted group, Pandemrix induced local reactions more often than FluvalP and than Focetria (51.2\% vs $27.6 \%$ and 
Table 1 Demographic, clinical characteristics and immunomodulatory treatments of 575 patients according to $\mathrm{H} 1 \mathrm{~N} 1$ vaccine group

\begin{tabular}{lllll}
\hline & & Non- & \\
& All & Adjuvanted & adjuvanted \\
patients & vaccines & vaccine & $p$ \\
$(N=575)$ & $(N=499)$ & $(N=76)$ & Value $\dagger$ \\
\hline
\end{tabular}

Demographic characteristics

Sex

Male, $n(\%)$

Age (years), mean (SD)

Pregnancy, $\mathrm{n}(\%)$

Clinical characteristics

IBD phenotype*

$$
\begin{aligned}
& \text { CD, n (\%) } \\
& \text { UC, n (\%) } \\
& \text { IC, n (\%) }
\end{aligned}
$$

Disease duration (years), median (IQR)

Age of diagnosis

$$
\text { A1 }
$$

A2

A3

Disease location

L1

L2

L3

L4

Disease behaviour

B1

B2

B3

$\mathrm{p}+$

Extent of UC

E1

E2

E3

Immunomodulatory treatments

Corticosteroids, $\mathrm{n}(\%)$

Azathioprine/6MP, $\mathrm{n}(\%)$

Methotrexate, $\mathrm{n}(\%)$

Anti-TNF therapies, $\mathrm{n}(\%)$

Infliximab, $\mathrm{n}(\%)$

Adalimumab, $\mathrm{n}(\%)$

Certolizumab, $\mathrm{n}(\%)$

Calcineurin inhibitors, $\mathrm{n}(\%)$

Others, $\mathrm{n}(\%)$

Monotherapy, n (\%)

Receipt of additional vaccines

H1N1 booster (2nd injection), $\mathrm{n}(\%) \quad \mathbf{2 1 2}(\mathbf{3 6 . 9 )} \quad 140(28.1) \quad 72$ (94.7) $\quad \mathbf{0 . 0 0 1}$ Seasonal influenza vaccine, $\mathrm{n}(\%) \quad \mathbf{3 2 8}(\mathbf{5 7 )} \quad 291(58.3) \quad 37(48.7) \quad \mathbf{0 . 1 1 4}$ Pneumococcal vaccine, $\mathrm{n}(\%) \quad \mathbf{7 0}(\mathbf{1 2 . 2 )} \quad 68(13.6) \quad 2(2.6) \quad \mathbf{0 . 0 0 6}$

Adjuvanted vaccines are Pandemrix, Focetria and FluvalP. Non-adjuvanted vaccine is Celvapan.

*According to Montreal classification.

†Depicts difference between adjuvanted vaccines and non-adjuvanted vaccine

$\mathrm{CD}$, Crohn's disease; IBD, inflammatory bowel disease; IC, indeterminate colitis; TNF, tumour necrosis factor; UC, ulcerative colitis; 6-MP, 6 mercaptopurine.

$15.4 \%, \mathrm{p}<0.0001)$. Patients with local reactions were younger than patients without (mean age 38.7 (SD 13.6) vs 41.2 (SD 14.1), $p=0.039$ ). Local symptoms were observed in $36.7 \%$ of patients aged below 50 years old and in $28.2 \%$ of patients above 50 years $(\mathrm{p}=0.06)$. Administration of seasonal influenza vaccine did not influence the rate of local symptoms due to H1N1 vaccine (data not shown). Patients who received seasonal vaccine and $\mathrm{H} 1 \mathrm{~N} 1$ vaccine on the same day tended to experience

\begin{tabular}{|c|c|c|c|c|}
\hline \multirow[b]{2}{*}{ Effect } & \multicolumn{3}{|c|}{ Adjuvanted vaccine } & \multirow{2}{*}{$\begin{array}{l}\begin{array}{l}\text { Non- } \\
\text { adjuvanted } \\
\text { vaccine }\end{array} \\
\text { Celvapan } \\
(\mathrm{N}=76)\end{array}$} \\
\hline & $\begin{array}{l}\text { Pandemrix } \\
\text { (AS03) } \\
(\mathrm{N}=295)\end{array}$ & $\begin{array}{l}\text { Focetria } \\
\text { (MF59) } \\
(\mathrm{N}=175)\end{array}$ & $\begin{array}{l}\text { FluvalP } \\
\text { (aluminium } \\
\text { phosphate) } \\
(\mathrm{N}=29)\end{array}$ & \\
\hline \multicolumn{5}{|l|}{ Local reaction } \\
\hline Any reaction, $\mathrm{n}(\%)$ & $160(54.2)$ & $28(16.0)$ & $8(27.6)$ & $3(3.9)$ \\
\hline Pain, n (\%) & $151(51.2)$ & $27(15.4)$ & $8(27.6)$ & $3(3.9)$ \\
\hline Redness, n (\%) & $26(8.8)$ & $4(2.3)$ & $0(0)$ & $0(0)$ \\
\hline Warmth, n (\%) & $10(3.4)$ & $0(0)$ & $0(0)$ & $0(0)$ \\
\hline Swelling, n (\%) & $33(11.2)$ & $2(1.1)$ & $0(0)$ & $0(0)$ \\
\hline \multicolumn{5}{|l|}{ Systemic reaction } \\
\hline Any reaction, n (\%) & $64(21.7)$ & $11(6.3)$ & $0(0)$ & $14(18.4)$ \\
\hline Shivering, n (\%) & $3(1.0)$ & $1(0.6)$ & $0(0)$ & $0(0)$ \\
\hline Fever $\geq 38^{\circ} \mathrm{C}, \mathrm{n}(\%)$ & $16(5.4)$ & $2(1.1)$ & $0(0)$ & $4(5.3)$ \\
\hline Unusual fatigue, n (\%) & $24(8.1)$ & $4(2.3)$ & $0(0)$ & $7(9.2)$ \\
\hline Malaise, n (\%) & $19(6.4)$ & $1(0.6)$ & $0(0)$ & $5(6.6)$ \\
\hline Headache, n (\%) & $17(5.8)$ & $3(1.7)$ & $0(0)$ & $1(1.3)$ \\
\hline Muscle pain, $\mathrm{n}(\%)$ & $15(5.1)$ & $3(1.7)$ & $0(0)$ & $0(0)$ \\
\hline Arthralgia, n (\%) & $5(1.7)$ & $1(0.6)$ & $0(0)$ & $1(1.3)$ \\
\hline Use of pain killers, $\mathrm{n}(\%)$ & $16(5.4)$ & $0(0)$ & $0(0)$ & $1(1.3)$ \\
\hline \multicolumn{5}{|c|}{ Increased bowel movements per day, diarrhoea and/or bloody diarrhoea } \\
\hline Any IBD, n (\%) & $10(3.4)$ & $5(2.9)$ & $0(0)$ & $7(9.2)$ \\
\hline Crohn's disease, $\mathrm{n}$ & 8 & 3 & 0 & 5 \\
\hline Ulcerative colitis, $n$ & 2 & 2 & 0 & 2 \\
\hline Indeterminate colitis, $\mathrm{n}$ & 0 & 0 & 0 & 0 \\
\hline
\end{tabular}
more local symptoms than patients who received separated
Table 2 Solicited local and systemic adverse effects within 7 days of receipt of first dose of adjuvanted or non-adjuvanted vaccines

$\mathrm{IBD}$, inflammatory bowel disease.

injections ( $45.5 \%$ vs $37.0 \%, \mathrm{p}=0.22)$. In multivariate analysis, after bootstrap resampling, the type of vaccine was the only factor related to the rate of local solicited reactions.

The most common systemic reaction was fatigue, reported by $35(6.1 \%)$ patients. No significant differences in the rate of systemic reaction were found between adjuvanted and nonadjuvanted vaccines, although they were more common in the Pandemrix group (21.7\% vs 18.4\%). A total of 17 (3.0\%) subjects reported use of pain killers. Twenty-two subjects (3.8\%) reported body temperature $>38^{\circ} \mathrm{C}$. No severe adverse side effects were noted. With the same subtype of vaccine, patients with $\mathrm{CD}$ and UC had similar rates of systemic symptoms except fatigue, which was more common in $\mathrm{CD}$ patients vaccinated with Pandemrix $(10.2 \%$ vs $1.6 \%, p=0.031)$. Administration of seasonal influenza vaccine at any time did not influence the rate of systemic symptoms due to $\mathrm{H} 1 \mathrm{~N} 1$ vaccine (data not shown). No association was found between rate of systemic symptoms and age $(\mathrm{p}=0.477)$.

Rates of any local or systemic solicited adverse events were not associated with any specific immunomodulatory treatment or biological therapy (data not shown). Moreover, there was no difference in the rates of local $(37.2 \%$ vs $33.6 \%, p=0.38)$ or systemic $(18.3 \%$ vs $14.4 \%, p=0.67)$ reactions in patients receiving monotherapy or combined therapy.

Effect of vaccination on IBD activity

Only $3.9 \%$ of patients (16 CD and six UC) had an increase in daily bowel movements and increased diarrhoea or bloody stools during the first week after H1N1vaccination. The reaction lasted for a median of 5 days and resolved spontaneously, except in one patient, who needed to increase corticosteroid dosage, and another, who was started on budesonide in addition to methotrexate. This phenomenon was observed with all types of 
vaccine except FluvalP, and was more common with Celvapan than with adjuvanted vaccines $(9.2 \%$ vs $3.0 \%, p=0.018)$. Of the 15 patients who received the adjuvanted vaccines, nine had symptoms after the first dose and six after the second dose, whereas all seven patients vaccinated with Celvapan, the non-adjuvanted vaccine, had symptoms after the first dose. Interestingly, six of these patients received a second dose of Celvapan and tolerated the second injection well. Immunomodulatory treatments or biological therapy did not influence the occurrence of such adverse reaction. CD and UC patients were similarly affected $(3.9 \%$ vs $3.8 \%, p=0.93)$. Of note, occurrence of these symptoms was independent of the age of patients.

Data on follow-up disease activity were available for 554 patients and are given in table 3 . Patients were scored at baseline and 4 weeks (median 31 days; IOR 28-37) after the last administration of $\mathrm{H} 1 \mathrm{~N} 1$ vaccine. The median disease activity score at baseline was 1 (IOR 0-3) and 1 (IOR 0-2) for CD and UC, respectively. Thirty-four CD patients $(8.7 \%)$ had a $\mathrm{HBI}$ score above 5 points, and 22 UC patients (13.9\%) had a PMS above 3 points. Four weeks after the last vaccination, absence of flare was observed in 377 patients with CD (96.7\%) and 151 with UC (95.6\%). Thirteen CD patients (3.3\%) had a median rise of 5 (IOR 4-7) points, and seven UC (4.4\%) patients had a median rise of 3 (IOR 3-5) points. Evolution of disease activity in patients with slightly higher scores at baseline was similar to those with the lowest score (data not shown). Increase in disease activity was not related to type of vaccine $(p=0.33)$ or type of IBD $(\mathrm{p}=0.20)$.

Of the 22 patients who experienced increased daily bowel movements, diarrhoea or bloody diarrhoea during the first week after vaccination, three of the 19 for whom data were available $(15.8 \%)$ had an increase of 3 or more points in their disease activity score 4 weeks after vaccination. UC patients were more likely than $\mathrm{CD}$ patients to keep an increased disease activity (one $\mathrm{CD}$ and two UC).

\section{DISCUSSION}

During last winter, the availability of vaccines against influenza H1N1v infection across Europe was variable. Most European countries used the adjuvanted forms of the vaccine, with composition and type of adjuvants varying from one vaccine to another. The use of adjuvanted and non-adjuvanted influenza vaccines in general had never been investigated in a large cohort of patients with IBD. The number of subjects included in the study allowed us to estimate the frequency of local and systemic reactions with good precision. The safety and tolerability profiles of adjuvanted and non-adjuvanted influenza vaccines were favourable in this large cohort of patients. In our study, more than half of patients with IBD additionally received the seasonal influenza vaccine according to the European recom-

Table 3 Evolution of clinical inflammatory bowel disease (IBD) score 4 weeks after vaccination

\begin{tabular}{lccl}
\hline IBD clinical score activity & $\mathbf{C D}(\mathbf{N}=\mathbf{3 9 0})$ & UC $(\mathbf{N}=\mathbf{1 5 8})$ & IC (N=6) \\
\hline HBI at baseline, median (IOR) & $1(0 ; 3)$ & - & - \\
PMS at baseline, median (IQR) & - & $1(0 ; 2)$ & $1(0 ; 2)$ \\
Absence of flare, $\mathrm{n}(\%)$ & $377(96.7)$ & $151(95.6)$ & $5(83.3)$ \\
Rise of 3 points, $\mathrm{n}(\%)$ & $1(0.2)$ & $4(2.5)$ & $0(0)$ \\
Rise of 4 points, $\mathrm{n}(\%)$ & $3(0.8)$ & $1(0.6)$ & $1(16.7)$ \\
Rise of $\geq 4$ points, $\mathrm{n}(\%)$ & $9(2.3)$ & $2(1.3)$ & $0(0)$
\end{tabular}

CD, Crohn's disease; HBI, Harvey-Bradshaw Index; IC, indeterminate colitis; PMS, Partial Mayo Score; UC, ulcerative colitis. mendations at the time of the pandemic. However, we observed a very low rate of pneumococcal vaccination despite recommendations and the well-known pneumococcal pneumonia complications that occur after influenza infection. Our study may underestimate the true percentage of pneumococcal vaccination, as some patients may have received this vaccine a few years before the study.

The side effect profile of $\mathrm{H} 1 \mathrm{~N} 1$ vaccines in immunocompromised patients with IBD, particularly the type of solicited adverse events, is consistent with previous reports in the immunocompetent population. ${ }^{2}$ The most common local symptom observed with all types of vaccine was pain at the injection site, as observed with other studies in immunocompetent subjects. ${ }^{2} 313$ Other local symptoms were otherwise rare. Systemic symptoms such as unusual fatigue, myalgia, headache and malaise were found similarly to previous reports in the immunocompetent population, although large variations exist and correlate mostly with the type of vaccine. This indicates that the reactions seen in patients with IBD are similar to those expected in the general population and are not influenced by either the inflammatory intestinal disease itself or any immunomodulatory treatment or biological therapy. However, direct comparison of the tolerance between immunocompetent and immunocompromised patients is not easy. We did not observe any association between any immunomodulatory treatment or biological therapy and the rate of local or systemic symptoms. As expected, administration of adjuvanted vaccines was associated with an increased incidence of solicited local reactions, which were all transient, as classically reported by previous studies. ${ }^{14-17}$ In multivariate analysis, only the type of vaccine correlated with rate of local symptoms. Surprisingly, we observed important variations in terms of solicited local reactions within the adjuvanted group. Pandemrix, Focetria and FluvalP differ in composition in terms of viral strain doses and the type of adjuvant used. This may account for these differences. We found, like others, ${ }^{13}$ that administration of seasonal influenza on the same day as the $\mathrm{H} 1 \mathrm{~N} 1$ vaccine led to more local symptoms. Systemic reactions were observed similarly in patients receiving adjuvanted or non-adjuvanted vaccines, although headache, arthralgia, muscle pain and use of pain killers were more common with Pandemrix than with non-adjuvanted vaccine. In trials comparing systemic symptoms in non-adjuvanted and MF59-adjuvanted vaccines, no significant difference in frequency or severity was observed. ${ }^{2}$ In our study, systemic reactions were observed in 89 of 575 patients (15.5\%). Observed rates of systemic symptoms were similar with adjuvanted and non-adjuvanted vaccines ( $15.0 \%$ vs $18.4 \%)$. The $95 \%$ CI for the difference was $-12 \%$ to $6 \%$, and therefore our trial may not have enough power to highlight differences between the adjuvanted and non-adjuvanted group. Gastrointestinal symptoms occurring within 7 days of vaccination were much more common with Celvapan than with adjuvanted vaccines. The European Medicines Agency indicated recently that gastrointestinal symptoms such as nausea, diarrhoea and abdominal pain were common $(\geq 1 / 100$ to $<1 / 10)$ with Celvapan and uncommon $(\geq 1 / 1000$ to $<1 / 100$ ) with Pandemrix or Focetria in non-IBD immunocompetent patients. ${ }^{18}$ These symptoms, also observed in our cohort, may therefore not be related to IBD but to the vaccine itself.

Regular concerns, particularly in patients with immunemediated inflammatory disease (IMID), arise around adjuvanted vaccines despite numerous data showing a good safety profile. ${ }^{19}$ Part of clinicians' concerns about the safety of vaccination in IMID originated from a number of case reports suggesting an effect of vaccination on IMID onset or course. ${ }^{20}$ These publications led to a belief among some clinicians that vaccination 
might trigger a flare of the underlying IMID. Live vaccines are generally contraindicated in immunocompromised patients, so reports dealing with their effect on disease activity are rare. In a relatively small retrospective study, measlesmumps-rubella booster vaccination in children with juvenile idiopathic arthritis appeared safe, as vaccination did not induce infection, nor did it significantly increase disease activity or medication use. ${ }^{21} 22$ For non-live vaccines, substantial literature data support the conclusion that immunisation of IMID patients does not increase clinical or laboratory parameters of disease activity. ${ }^{23}$ Most of this evidence comes from mediumsized controlled trials in which disease activity was mostly assessed from general clinical symptoms and pain scores. Some studies also used standardised clinical disease activity scores such as the Systemic Lupus Erythematosus Disease Activity Index ${ }^{24}$ and the Disease Activity Score in rheumatoid arthritis. ${ }^{25} \mathrm{We}$ provide new information on the effect of adjuvanted and nonadjuvanted influenza vaccines on the IBD activity. Two studies have shown the effect of influenza vaccination in 51 and 146 paediatric and young adult patients with IBD. They did not show modification of disease activity after administration of trivalent influenza non-adjuvanted vaccine. ${ }^{26} 27$ Although imperfect, clinical scores such as HBI and PMS are useful tools in clinical trials. In our cohort, before vaccination, all patients had a stable inflammatory disease. This was left to the physician discretion, but was also well reflected by the low HBI and PMS values obtained at baseline. In $\mathrm{CD}$, a decrease of 3 points in the HBI score is recognised as response to therapy. ${ }^{8}$ In previous studies, it has been shown that the PMS performed as well as the full Mayo Score in identifying clinical response. ${ }^{9}$ However, criteria for defining a response in UC is less clear, as authors identify a change of $2,2.5$ or 3 points in the PMS as a significant response. ${ }^{9}{ }^{11}$ Hence, in our study, an increase of 3 points for either HBI or PMS was considered a significant flare. With these criteria, only a minority of patients had an increased score 4 weeks after vaccination. In these patients, the flare up was not related to either type of IBD or type of vaccine administered. If an increase of 2 points in the PMS is considered as clinically significant, the percentage of UC patients with a stable disease 4 weeks after $\mathrm{H} 1 \mathrm{~N} 1$ vaccination falls slightly from $95.6 \%$ to $93.7 \%$. Therefore, no matter the criteria used to define clinical flare in UC, in the vast majority of patients with UC, disease activity appears to remain stable after vaccination. Taken together, our results indicate that the risk/benefit ratio of influenza vaccination in patients with IBD treated with immunomodulators and biological therapy is probably highly in favour of a vaccination strategy.

There are some limitations of this study. The main one concerns the assessment of HBI and PMS by phone 4 weeks after the vaccine. Physical confirmation was not possible, but we believe that the effect of this limitation is low. Indeed, when a large modification of the physical examination occurs, it is often accompanied by new symptoms, and we assume that significant modification had therefore been reported. However, use of phone records may lead to underestimation or overestimation of these clinical scores. The second limitation concerns the confounding bias (unrelated to vaccine administration) regarding an increased score 4 weeks after receipt of the vaccine. We cannot rule out the possibility of drugs, non-steroidal anti-inflammatory drugs, viral or bacterial digestive infection, or stress being responsible for increasing the score 4 weeks after vaccination.

In conclusion, our results support the good safety profile of adjuvanted and non-adjuvanted influenza vaccine in patients with IBD and bring new evidence that use of adjuvanted vaccine has few or no effects on IBD activity. Nevertheless, definitive conclusions can only be derived from well-conducted, randomised, placebo controlled trials or large registries. However, this study provides evidence that adjuvanted and non-adjuvanted influenza vaccines are unlikely to induce re-activation in patients with IBD and will help us to manage large distributions of adjuvanted or non-adjuvanted vaccines in the case of further pandemics

\section{Author affiliations}

${ }^{1}$ Gastroenterology Unit, Cliniques Universitaires UCL Mont-Godinne, Yvoir, Belgium ${ }^{2}$ Gastroenterology Unit, Medical University Vienna, Vienna, Austria

${ }^{3}$ Biostatistics Unit, Université Lille Nord de France, CHUR Lille, France

${ }^{4}$ Gastroenterology Unit, Hull Royal Infirmary, Hull, UK

${ }^{5}$ Gastroenterology Unit, Universita Cattolica del Sacro Cuore, Roma, Italy

${ }^{6}$ Gastroenterology Unit, Université Henri Poincaré 1, CHU de Nancy, France

${ }^{7}$ Gastroenterology Unit, H Reina Sofia, Cordoba, Spain

${ }^{8}$ Gastroenterology Unit, University of Messina, Messina, Italy

${ }^{9}$ Gastroenterology Unit, VU University Medical Center, Amsterdam, The Netherlands

${ }^{10}$ Gastroenterology Unit, University of Szeged, Szeged, Hungary

${ }^{11}$ Gastroenterology Unit, Kennemer Gasthuis, Haarlem, Netherlands

${ }^{12}$ Gastroenterology Unit, Helsinki University Central Hospital, Helsinki, Finland

${ }^{13}$ Gastroenterology Unit, Mater Dei Hospital, Msida, Malta

${ }^{14}$ Gastroenterology Unit, Liège University and CHU Liège, Belgium

${ }^{15}$ Gastroenterology Unit, St Marks Hospital, London, UK

${ }^{16}$ Gastroenterology Unit, Chaim Sheba Tel Hashomer Medical Center, Ramat Gan, Israel

${ }^{17}$ Gastroenterology Unit, Charité - Universtiätsmedizin Berlin, Germany

${ }^{18}$ Gastroenterology Unit, Rambam Human Health Care Campus, Haifa, Israel

${ }^{19}$ Gastroenterology Unit, IBD Unit, IRCCS Humanitas, Rozzano, Milan, Italy

${ }^{20}$ Service Universitaire des Maladies Infectieuses et du Voyageur, CHU Tourcoing and ATIP-Avenir INSERM U995, Tourcoing, France

${ }^{21}$ Gastroenterology Unit, La Princesa and Centro de Investigación Biomédica en Red de Enfermedades Hepáticas y Digestivas (CIBERehd), Madrid, Spain

Acknowledgements We acknowledge scientific unit support from the Cliniques Universitaires UCL Mont-Godinne, particularly Mme Michèle Duclos, for database management. We are grateful to Jean-Frédéric Colombel, Simon Travis, Walter Reinisch and Séverine Vermeire for support and guidance throughout the project.

Competing interests J-FR received lecture fees from speaking at continuing medical education events from Abbott Laboratories and Schering-Plough and paid advisory board for Glaxo Smithkline. PP received consulting fees and speaker fees from Abbott Laboratories; LP-B has received consulting fees from Abbott Laboratories and UCB Pharma. VG received lecture fees from speaking at continuing medical education events from Abbott Laboratories and MSD. WF received a research grant from Schering-Plough TS received lecture fees from speaking at continuing education events from Abbott, MSD and Tillotts Pharma. EL received research grants from MSD, AstraZeneca and Abbott, speaker fees from Abbott, AstraZeneca, Ferring, Falk, MSD/Schering Plough, Menarini, Movetis and Nycomed and he served as a consultant or advisor for Abbott, AstraZeneca, MSD/Schering Plough, Millenium, Ferring, Shire. JM has served as a consultant for Schering Plough and has received speaker fees from Abbott. YY has received travel grants, honoraria for presentation at workshops and consultancy honoraria from Bristol-Myers Squibb, Boehringer Ingelheim, Gilead, Glaxo-SmithKline, Merck, Pfizer, Roche and Tibotec. BB received speaker honoraria from Abbott. TH received honoraria from Abbott and MSD. RP received honoraria from Abbott and MSD.

\section{Patient consent Obtained.}

Ethics approval This study was conducted with the approval of the UCL Mont Godinne University Hospital, Yvoir, Belgium.

Contributors J-FR and PP were involved in the study concept and design and writing the manuscript. JS performed all statistical analyses. LP-B and EL were involved in writing the manuscript. J-FR, PP, SS, MM, LP-B, VG, WF, DPvanA, KF, NKHdeB, TS $P E, E L, S P, U K, J M, B M, G F$ and $M C$ were all involved in acquisition of data. J-FR had full access to all of the data and takes full responsibility for the veracity of the data and analysis.

In addition to the authors, the following investigators participated in the study: Rita Monterubbianesi (S Camillo-Forlanini Hospital, Rome, Italy), Benedikt Blaha

(Wilhelminenspital, Vienna, Austria), Monica Cesarini (University of Rome Sapienza, Policlinico Umberto, Rome, Italy), Thomas Haas (Paracelsus Medical University, Salzburg, Austria) and Reingard Platzer (Landesklinikum, Wiener Neustadt, Austria). Alfredo Papa (Universita Cattolica del Sacro Cuore Rome, Italy), Gianluca Andrisani (Universita Cattolica del Sacro Cuore Rome, Italy), Naila Arebi (St Marks Hospital London UK), Javier P Gisbert (La Princesa and CIBERehd Madrid, Spain).

Provenance and peer review Not commissioned; externally peer reviewed. 


\section{REFERENCES}

1. Ginsberg M, Hopkins J, Maroufi A, et al. Swine influenza A (H1N1) infection in two children-Southern California, March-April 2009. MMWR Morb Mortal Wkly Rep 2009;58:400-2

2. Clark TW, Pareek M, Hoschler K, et al. Trial of 2009 influenza A (H1N1) monovalent MF59-adjuvanted vaccine. N Engl J Med 2009;361:2424-35.

3. Greenberg ME, Lai MH, Hartel GF, et al. Response to a monovalent 2009 influenza A (H1N1) vaccine. N Engl J Med 2009;361:2405-13.

4. Rahier JF, Yazdanpanah Y, Viget N, et al. Review article: influenza A (H1N1) virus in patients with inflammatory bowel disease. Aliment Pharmacol Ther 2010;31:5-10.

5. Harvey RF, Bradshaw JM. A simple index of Crohn's-disease activity. Lancet 1980;1:514.

6. Schroeder KW, Tremaine WJ, Ilstrup DM. Coated oral 5-aminosalicylic acid therapy for mildly to moderately active ulcerative colitis. A randomized study. N Engl J Med 1987; 317:1625-9.

7. Silverberg MS, Satsangi J, Ahmad T, et al. Toward an integrated clinical, molecular and serological classification of inflammatory bowel disease: report of a Working Party of the 2005 Montreal World Congress of Gastroenterology. Can J Gastroenterol 2005;19(Suppl A):5-36.

8. Best WR. Predicting the Crohn's disease activity index from the Harvey-Bradshaw Index. Inflamm Bowel Dis 2006:12:304-10.

9. Lewis JD, Chuai S, Nessel L, et al. Use of the noninvasive components of the Mayo score to assess clinical response in ulcerative colitis. Inflamm Bowel Dis 2008:14:1660-6.

10. Vermeire S, Schreiber S, Sandborn WJ, et al. Correlation between the Crohn's disease activity and Harvey-Bradshaw indices in assessing Crohn's disease severity. Clin Gastroenterol Hepatol 2010;8:357-63.

11. Rutgeerts $\mathbf{P}$, Sandborn WJ, Feagan BG, et al. Infliximab for induction and maintenance therapy for ulcerative colitis. N Engl J Med 2005;353:2462-76.

12. Sauerbrei W, Schumacher M. A bootstrap resampling procedure for model building application to the Cox regression model. Stat Med 1992;11:2093-109.

13. Vajo Z, Tamas F, Sinka L, et al. Safety and immunogenicity of a 2009 pandemic influenza A H1N1 vaccine when administered alone or simultaneously with the seasonal influenza vaccine for the 2009-10 influenza season: a multicentre, randomised controlled trial. Lancet 2010:375:49-55.
14. Treanor JJ, Campbell JD, Zangwill KM, et al. Safety and immunogenicity of an inactivated subvirion influenza $A$ (H5N1) vaccine. $N$ Engl J Med 2006:354:1343-51.

15. Podda A. The adjuvanted influenza vaccines with novel adjuvants: experience with the MF59-adjuvanted vaccine. Vaccine 2001;19:2673-80

16. De Donato $\mathbf{S}$, Granoff D, Minutello $\mathrm{M}$, et al. Safety and immunogenicity of MF59-adjuvanted influenza vaccine in the elderly. Vaccine 1999;17:3094-101.

17. Gasparini R, Pozzi T, Montomoli E, et al. Increased immunogenicity of the MF59-adjuvanted influenza vaccine compared to a conventional subunit vaccine in elderly subjects. Eur J Epidemiol 2001;17:135-40.

18. http://www.ema.europa.eu/ema/index (accessed 21 Nov 2010).

19. Pellegrini M, Nicolay U, Lindert K, et al. MF59-adjuvanted versus non-adjuvanted influenza vaccines: integrated analysis from a large safety database. Vaccine 2009;27:6959-65.

20. Conti F, Rezai S, Valesini G. Vaccination and autoimmune rheumatic diseases. Autoimmun Rev 2008;8:124-8.

21. Heijstek MW, Pileggi GC, Zonneveld-Huijssoon E, et al. Safety of measles, mumps and rubella vaccination in juvenile idiopathic arthritis. Ann Rheum Dis 2007:66:1384-7

22. Borte S, Liebert UG, Borte $\mathrm{M}$, et al. Efficacy of measles, mumps and rubella revaccination in children with juvenile idiopathic arthritis treated with methotrexate and etanercept. Rheumatology (Oxford) 2009;48:144-8.

23. Rahier JF, Moutschen M, Van Gompel A, et al. Vaccinations in patients with immune-mediated inflammatory diseases. Rheumatology (Oxford) 2010;49:1815-27.

24. Del Porto $\mathbf{F}$, Lagana B, Biselli R, et al. Influenza vaccine administration in patients with systemic lupus erythematosus and rheumatoid arthritis. Safety and immunogenicity. Vaccine 2006;24:3217-23.

25. Fomin I, Caspi D, Levy V, et al. Vaccination against influenza in rheumatoid arthritis: the effect of disease modifying drugs, including TNF alpha blockers. Ann Rheum Dis 2006;65:191-4.

26. Lu Y, Jacobson DL, Ashworth LA, et al. Immune response to influenza vaccine in children with inflammatory bowel disease. Am J Gastroenterol 2009;104:444-53.

27. Mamula $\mathbf{P}$, Markowitz JE, Piccoli DA, et al. Immune response to influenza vaccine in pediatric patients with inflammatory bowel disease. Clin Gastroenterol Hepatol 2007:5:851-6.

\section{Have confidence in your decision making.}
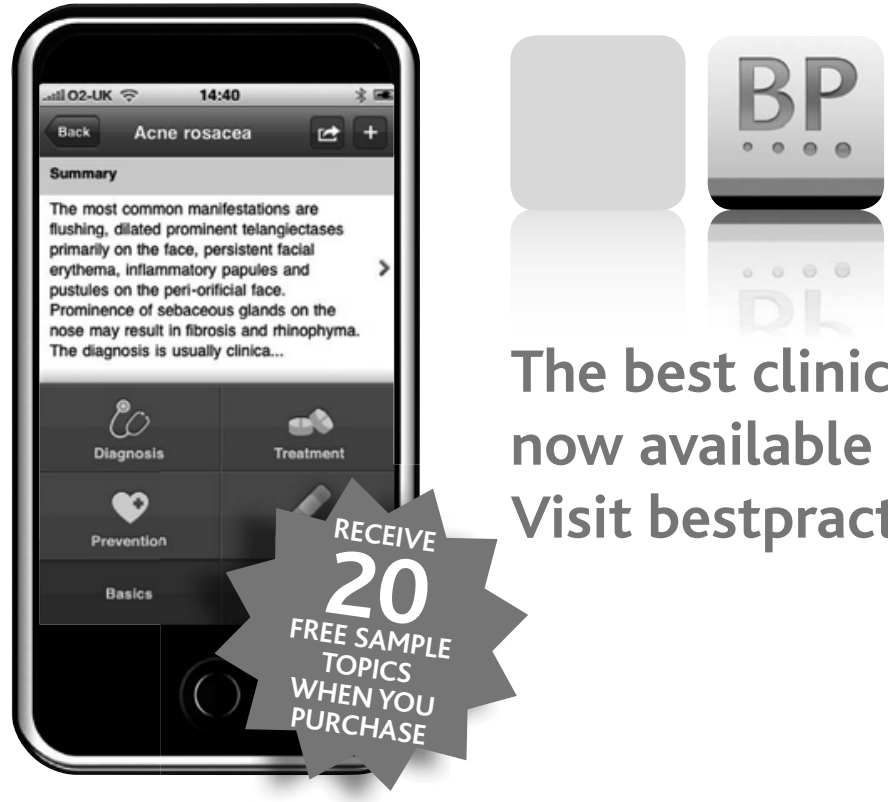

The best clinical decision support tool is now available as an app for your iPhone. Visit bestpractice.bmj.com/app

\section{clinicians • medical students • nurses • healthcare practitioners}

